\title{
PERCEPCIÓN DE LOS PADRES SOBRE EL EMPODERAMIENTO DIGITAL DE LAS FAMILIAS EN HOGARES HIPERCONECTADOS
}

\section{The perception of parents about the digital} empowerment of family in hyperconnected households

\author{
Teresa Torrecillas-Lacave, Tamara Vázquez-Barrio y Laura Monteagudo- \\ Barandalla
}

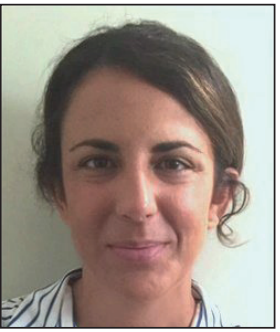

Teresa Torrecillas-Lacave, doctora en periodismo por la Universidad San Pablo CEU, es secretaria académica del Programa Internacional de Doctorado en Comunicación Social, de la CEU Escuela Internacional de Doctorado (Ceindo). Sus principales líneas de investigación son la comunicación y los públicos vulnerables (concretamente, menores), nuevos medios y alfabetización mediática. Ha participado en proyectos competitivos y en la actualidad es investigadora principal junto a Luis Núñez-Ladevéze del proyecto Auctoritas doméstica, capacitación digital y comunidad de aprendizaje en familias con menores escolarizados, financiado por el Ministerio de Economía y Competitividad (CSO 2013-42166-R).

http://orcid.org/0000-0001-6167-658X

Universidad San Pablo CEU Paseo Juan XXIII, 10. 28040 Madrid, España teresat@ceu.es



Tamara Vázquez-Barrio es profesora de opinión pública en la Universidad CEU San Pablo. Licenciada en Comunicación audiovisual y doctora en Periodismo por la misma universidad, tiene un master en Producción audiovisual (UCM) y el título de Experto universitario en diseño y tratamiento estadístico de estudios de mercado por la UNED. Desarrolla dos líneas de investigación: sobre los efectos de los medios de comunicación en la audiencia infantil y juvenil, con especial interés en la alfabetización digital, la mediación familiar y la responsabilidad social de los medios; y sobre comunicación política.

http://orcid.org/0000-0003-2789-8554

Universidad San Pablo CEU Paseo Juan XXIII, 10. 28040 Madrid, España tamarav@ceu.es

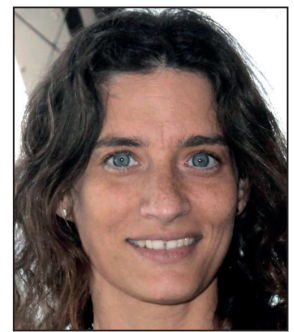

Laura Monteagudo-Barandalla es profesora e investigadora en diversas instituciones: Universidad CEU San Pablo, EUDE Business School, EUSchool y Esden Business School. Es licenciada en Administración y dirección de Empresas, con especialidad en Marketing e investigación de mercados por la Universidad Complutense de Madrid. Master en Marketing y publicidad digital, por la Universidad Antonio de Nebrija y doctorando en Comunicación especializada, innovación y tecnologías por la Universidad San Pablo-CEU. Especializada en marketing y en e-commerce, ha trabajado en investigación de mercados, agencias de publicidad como Young\&Rubicam y Contrapunto, y desarrollando proyectos de emprendimiento como la creación de dos e-commerce.

http://orcid.org/0000-0003-2956-0581

EUDE Business School y Universidad San Pablo CEU Arturo Soria, 245. 28033 Madrid, España lau.monteagudo@ceindo.ceu.es

\section{Resumen}

En este artículo se difunden los resultados de una investigación cualitativa encaminada a estudiar el hogar como escenario de comunicación multipantalla en el que se produce la inclusión digital de los hijos, y de los padres como agentes de mediación en el uso que los menores hacen de los servicios digitales. La técnica utilizada ha sido el grupo de discusión de padres con hijos escolarizados en la Comunidad de Madrid. Los resultados indican que estos hogares tienden a ser escenarios 
hiperconectados, con acceso temprano e intensivo de TIC, en los que no existe brecha digital de acceso y sí de uso. En tales hogares la tecnología favorece la cohesión familiar por la emergencia de nuevos espacios de comunicación digitales y en los que se ejerce cierta mediación con medidas de control del uso y consumo que hacen los menores de las TIC.

\title{
Palabras clave
}

Nuevas tecnologías; Servicios digitales; Internet; Educación mediática; Familias; Mediación parental; Alfabetización mediática; Medios de comunicación.

\begin{abstract}
In this article we disseminate the results of a qualitative research project aimed at studying the household as a multi-device communication setting in which both children and parents are involved in a digital environment where parents act as mediators in the use of digital services by minors. The methodology used was the so-called "discussion group" technique, that involved parents whose children attend school in the Autonomous Region of Madrid. The results show that these households are hyper-connected settings that offer early access and intense use of IT devices in which both parents and children have equal access to technology but use it in different ways. In these households, technology facilitates family cohesion as a result of the emergence of new forms of digital communication, and in which certain mediation occurs as parents take measures to control the use and exposure to technology by minors.
\end{abstract}

\section{Keywords}

New technologies; Digital services; Internet; Media education; Families; Parental mediation; Media literacy; Media.

Torrecillas-Lacave, Teresa; Vázquez-Barrio, Tamara; Monteagudo-Barandalla, Laura (2017). "Percepción de los padres sobre el empoderamiento digital de las familias en hogares hiperconectados". El profesional de la información, v. 26, n. 1, pp. 97-104.

https://doi.org/10.3145/epi.2017.ene.10

\section{Introducción y estado de la cuestión}

Los datos sobre la transformación digital de la sociedad española (Fundación Telefónica, 2015; Barómetro CIS, 2016) muestran la construcción de hogares multipantalla y nuevos hábitos de comunicación caracterizados por un alto componente social. Esta situación plantea un reto sin precedentes a los padres: cómo participar en la educación digital de los hijos favoreciendo hogares multipantalla responsables, que gestionen adecuadamente las oportunidades que ofrece internet y que eviten sus riesgos (Livingstone et al., 2011).

En este artículo difundimos resultados de la fase cualitativa del proyecto de investigación Auctoritas doméstica, capacitación digital y comunidad de aprendizaje en familias con menores escolarizados, cuyo objetivo ha sido obtener información sobre la percepción y actitud de los padres acerca de la inclusión digital de sus hogares. En esta fase de la investigación hemos tratado de responder al siguiente objetivo concreto: conocer y analizar las ideas de los padres sobre:

- características de los hogares como escenarios de consumo;

- internet y servicios digitales;

- competencias digitales, mediación y riesgos;

- internet como posible generador de nuevos espacios de comunicación intrafamiliares.

La hipótesis de investigación es que las ideas y cogniciones sobre internet y los servicios digitales que sostienen los padres pueden condicionar la construcción del hogar como escenario educativo en el uso y consumo de TIC por parte de los menores y el tipo de interacciones educativas de padres e hijos -mediación parental-; y por lo tanto pueden generar distintas formas de recepción multipantalla por parte de los menores escolarizados.
Los resultados de esta investigación son una aportación a los estudios de la recepción desde el enfoque ecológicosistémico (Broffenbrenner, 1987), en el que se fundamenta nuestro planteamiento. Este enfoque pone el acento en la importancia del contexto familiar para el desarrollo de los niños, considerado el escenario educativo más importante en su crecimiento. Como señala Muñoz-Silva (2005), "esto es debido a que las influencias familiares son las primeras y las más persistentes".

\section{Las ideas y cogniciones sobre internet y} los servicios digitales que sostienen los padres pueden condicionar la construcción del hogar como escenario educativo

Los padres son quienes diseñan el ambiente en el que los menores realizan sus actividades cotidianas -como es el uso de servicios digitales-, además de mediar y supervisar con el objeto de fomentar la autorregulación en los menores (Bradley, 1995; Rodrigo; Palacios, 1998).

En el hogar como contexto de recepción multipantalla, la mediación familiar la entendemos como instancia cultural desde donde los menores producen y se apropian de los sentidos y significaciones de los nuevos procesos comunicativos y que configura tanto la interacción con los nuevos medios -consumo y producción- como la creación del sentido de la interacción (Martín-Barbero, 1987; Orozco-Gómez, 1996). La relación causal familia, menores y nuevos medios, está perfectamente asumida por la comunidad científica (Livingstone; Bober, 2006). 
En los últimos años se están llevando a cabo muchos estudios desde esta perspectiva en el ámbito europeo (Livingstone; Haddon, 2008; García-Jiménez, 2012; Ruiz-San-Román; Ortiz-Sobrino; Porto-Pedrosa, 2013). A diferencia de lo que sucede con la televisión, en contextos multipantalla hay poca acumulación de conocimiento y están centrados en evaluar estrategias de mediación y su incidencia en el comportamiento digital de los hijos (Pérez-Sánchez, 2013; Airebe; Orozo; Medrano, 2014; Garmendia-Larrañaga et al., 2011).

Hay autores que consideran perjudiciales las estrategias de control porque cohíben la autonomía del niño (Staksrud; Livingstone, 2009), frente a otros que apuestan por tutelar el uso y consumo con ayudas técnicas y normas (Bringué-Sala; Sádaba-Chalezquer, 2008).

La mayoría de estas investigaciones están centradas en lo que algunos investigadores denominan componentes físicos, es decir, "aspectos observables como actores presentes en el uso y consumo de TIC o lugares de uso" (Padilla et al., 2015).

Nosotros nos sumamos a una línea más reciente y poco explorada, sobre todo en España, encaminada al análisis de los componentes actitudinales (Valkenburg; Soeters, 2001); es decir, ideas o cogniciones de las audiencias interactivas, como la percepción que tienen los padres sobre las TIC o su responsabilidad mediadora desde una perspectiva cualitativa, como condicionantes del grado y forma del tipo de intervención educativa que ejercen. Como señalan Álvarez et al. (2013), "las actitudes y dimensiones de los padres difieren según su edad y educación".

\section{Los hogares urbanos con menores es- colarizados tienden a ser hogares hiper- conectados con una fuerte presencia de dispositivos tecnológicos}

\section{Material y métodos}

Se han realizado dos grupos de discusión con padres con menores escolarizados de la Comunidad de Madrid. La decisión metodológica estuvo determinada por el carácter de esta fase de la investigación: un estudio de la recepción multipantalla que tiene por objeto entender los procesos que viven las audiencias interactivas en su relación con el medio digital (Callejo, 2001).

Para el desarrollo de los grupos el equipo investigador elaboró una guía de discusión a partir de los resultados previos obtenidos en la fase cuantitativa mediante encuesta.

El universo de la muestra está constituido por familias urbanas con menores escolarizados de la Comunidad de Madrid. Los ejes utilizados para la selección de la muestra estructural han sido:

- edad de los hijos;

- edad de los padres;

- sexo de los hijos.

Los resultados de la fase cuantitativa y de otros estudios señalados en el marco teórico (Álvarez et al., 2013) ponen de manifiesto que:
- la edad de los hijos condiciona la frecuencia de uso y, por tanto, el grado de exposición al medio;

- la edad de los padres y el sexo de los hijos pueden implicar diferentes percepciones y, por tanto, tipos de mediación.

Se han tenido en cuenta asimismo otros dos ejes estructuradores:

- estatus socioeconómico, a partir del nivel de formación de los padres y el tipo de trabajo (medio-alto, medio-medio y medio bajo);

- tipo de colegio (público o privado/concertado).

Pero no se han considerado predominantes porque al estar el universo limitado a familias urbanas de la Comunidad de Madrid se previó que no iban a proporcionar opuestos muy significativos.

El diseño muestral intencional es el siguiente:

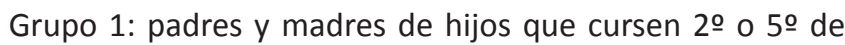
primaria.

Grupo 2: padres y madres de hijos que cursen 2ㅇ de ESO (educación secundaria obligatoria) o 1ㅇ de bachillerato.

Las características de cada grupo son las mismas:

- edad: mayores y menores de 45 años al 50\%;

- niños/niñas: padres/madres de hijos y de hijas al 50\%;

- sexo: hombres y mujeres al 50\%;

- titularidad del colegio: público y privado/concertado al $50 \%$;

- estatus socioeconómico: $13 \%$ clase media alta; $50 \%$ clase media-media y $38 \%$ clase media baja.

Cada grupo está compuesto por 8 individuos. No se ha buscado una proporción adecuada y estadística entre la muestra y el universo, sino que los principales sectores de la población que son objeto de estudio estén presentes, con sus criterios, comportamientos y discursos.

Hemos utilizado un criterio de representatividad sustantiva con el objeto de cubrir la variedad de las situaciones sociales, más que el reproducir sobre una escala reducida las características de la población. El punto de partida es el individuo; el criterio está centrado en el sujeto y no en las variables.

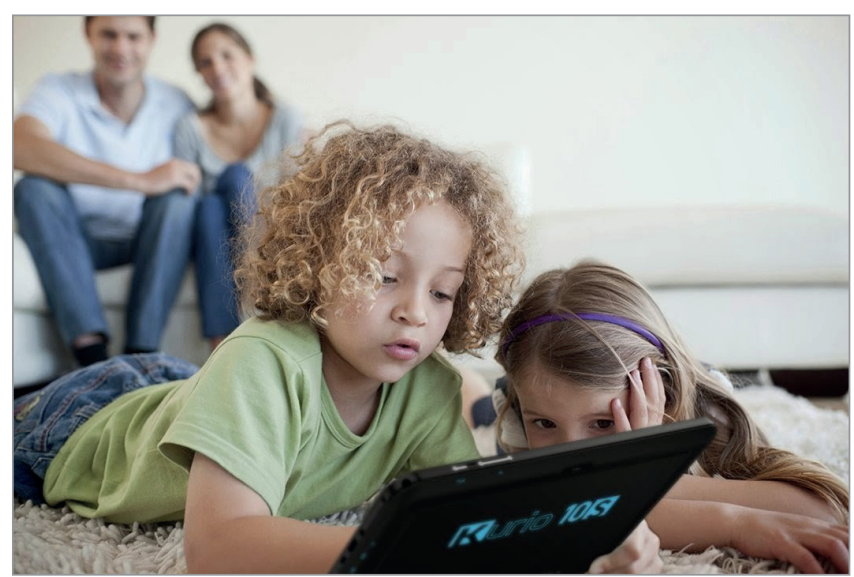

http://bd-dvd-copying-ripping.blogspot.com.es/2014/04/play-dvdmovies-on-kurio-10s.htm 


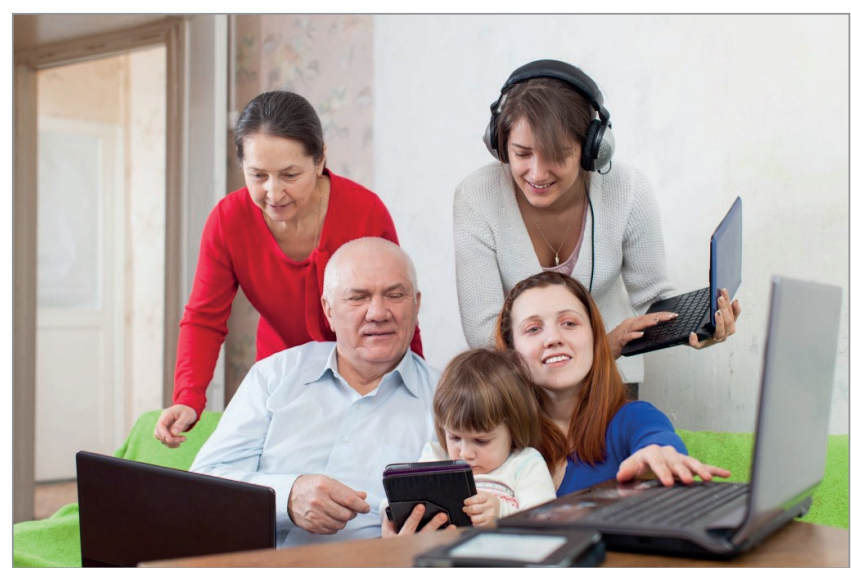

http://www.wintterlaw.com/digital-assets-to-include-in-your-estateplanning

Los grupos se llevaron a cabo el 22 de junio de 2016, tuvieron una duración de 2 horas, fueron visionados en directo por los técnicos participantes, grabados, las conversaciones fueron transcritas y toda la información procesada en fichas de procesamiento general y local y posteriormente analizada.

\section{Resultados}

\subsection{El hogar como escenario de comunicación mul- tipantalla}

Las características del escenario de comunicación -determinado por el grado de equipamiento tecnológico y por el lugar que ocupa éste en el espacio- son muy importantes porque reflejan el lugar simbólico que ocupan las tecnologías en las familias y porque fijan las posibilidades de acceso y uso por parte de los menores.

\section{La percepción de los riesgos descritos no} impide que los padres faciliten el acceso de los hijos a los dispositivos tecnológicos

Se puede afirmar que los hogares urbanos con menores escolarizados tienden a ser hogares hiperconectados con una fuerte presencia de dispositivos tecnológicos tanto por su situación predominante en el escenario como por diversidad y número.

Los dispositivos predominantes son tablets y móviles, seguidos de ordenadores de sobremesa, portátiles y videoconsolas de juegos. Los libros electrónicos y las smart-TV son las menos frecuentes.

El dispositivo por excelencia con mayor poder de atracción para todos los miembros de la familia es la tablet. La versatilidad de usos, facilidad e intuición en el manejo hace que sea accesible a todos los miembros independientemente de la edad o la formación. Se trata del dispositivo que los hijos adquieren a edades más tempranas (a partir de los 3 años).

La tablet es muy atractiva para los menores porque aúna prestaciones de otros dispositivos. Su uso conectado a la red wifi del hogar permite a los menores:
- seguir los canales infantiles de televisión a demanda;

- ver películas y series infantiles en plataformas como Yomvi;

- escuchar música y ver videoclips a través de YouTube;

- jugar solos o en red (alrededor de los 11 años).

Con los años, el uso de las tablets se va incorporando al mundo escolar convirtiéndose en una herramienta útil como libro electrónico y buscador de información.

El uso por parte de padres de las tablets se centra sobre todo en el seguimiento de series, lecturas de periódicos y libros, correo electrónico y operaciones bancarias, así como la ejecución de compras y cursos, planificación de viajes, almacenamiento de fotos, además de la búsqueda de información a través de Google y Google Maps. Valoran especialmente la portabilidad del dispositivo. Se tiende a tener más de una tablet en aquellos hogares con más de un hijo.

El smartphone es otro dispositivo prioritario en hogares hiperconectados fundamentalmente en la pre-adolescencia y adolescencia y también para los padres. Es habitual que en los hogares cada miembro disponga de un terminal móvil; pero éste difiere en prestaciones y usos en función de la edad y el uso que cumpla en cada momento vital.

\section{Los padres ven las TIC como una exten- sión del cordón umbilical, una herra- mienta escolar o como un medio de ne- gociación y estímulo en caso de conflicto con los hijos}

En menores de 10-12 años la mayoría de los terminales son heredados. Suelen usarse dentro del hogar, casi siempre conectados a la red wifi para descargas de juegos, música y vídeos en YouTube; pero no suelen contar con tarifas de datos y llamadas. Es a partir de los 12 años, cuando se suele activar un número móvil o contratar una tarifa de datos y llamadas. Se alude a la necesidad de estar localizables en caso de emergencia.

"Pienso que un niño de 10 años no tiene que tener móvil. Ya cuando empiecen a salir con 12-14 me interesa que lleve un móvil sí, que si tiene un problema me pueda localizar, que si necesita algo... No es que yo le controle a él, es que tenga un medio de acceso para comunicarse, nosotros antiguamente salíamos y tenías cabinas de teléfono pero ahora no hay" (G1).

En secundaria el móvil adquiere una mayor importancia al ser un dispositivo de comunicación no sólo con la familia sino también con el 'grupo de iguales'. Whatsapp es la principal aplicación para comunicarse entre los estudiantes de secundaria con sus padres, comunicación que se irá intensificando en edades avanzadas.

"Cuando tiene más tiempo sí que está con el rollo del WhatsApp, ellos todo el día comunicándose, ese es el tema, hay un tiempo que a lo mejor está de más... Pero sí he notado que va como a más. En cuanto a juegos no es mucho de ellos ya" (G2). 
El uso adulto de los móviles por parte de padres y madres se ha visto también intensificado desde la llegada de los smartphones y la tarifa de datos que ha provocado que aplicaciones como Whatsapp y Facebook creen cierta adicción a estar permanentemente comunicados y accesibles, sobre todo, con familiares y amigos. Otros usos habituales de los móviles por parte de padres y madres son similares a los realizados con la tablet: correo, operaciones bancarias, etc.

Se podría decir que el único dispositivo tecnológico que se suele compartir entre padres e hijos es el ordenador de sobremesa o portátil. Su uso se restringe al ámbito profesional en el caso de los padres y al educativo en el de los hijos. De hecho, solamente las tareas formativas reúnen a padres e hijos delante del ordenador.

"Yo por ejemplo, el pequeño, en el colegio el profesor tiene su blog, los libros de texto vienen con un código que entras en la editorial y hay actividades del curso en el que está con el libro, sobre todo, también los libros de inglés y sí que lo hacemos con ellos" (G1).

No existe brecha digital pero padres e hijos coinciden poco en la Red y tienen experiencias mediáticas diferentes

\subsection{Percepción y actitudes hacia internet y los servi- cios digitales}

Para los padres de la muestra, las TIC representan un avance en el conocimiento y desarrollo de sus hijos ya que les permiten acceder a la información y conocimiento de una forma más rápida, cómoda y lúdica cuando son pequeños.

"Ayuda al intelecto de los niños pero todo usado con medida, con una lógica. Con la tablet ya pueden empezar a programar y todas esas cosas están desarrollando el intelecto del niño. De forma inconsciente jugando aprenden. Es como el habla que los niños han aprendido hablando y escuchando, el tema de la tecnología es exactamente igual, están aprendiendo a base de usarlo" (G1).

Otra razón es que los padres las consideran fundamentales para que los hijos adquieran habilidades tecnológicas necesarias para competir en el futuro mercado laboral.

No obstante, en términos de comunicación familiar el acceso permanente a las TIC en el que crecen y se desarrollan los hijos se valora de forma ambivalente.

Por un lado existe cierto grado de conflicto familiar al existir la consciencia del aislamiento e individualismo que implica el uso personal y autónomo de estos dispositivos.

"Lo veo una barrera de comunicación, mientras están atentos a la pantalla no están hablando conmigo" (G1).

Por otro lado las TIC sirven a la cohesión familiar permitiendo la comunicación intrafamiliar de forma inmediata.

"Se ha perdido el cara a cara pero se ha transformado con otros medios. Yo me acuerdo cuando yo llegaba del colegio a mis padres les contaba todo, mis hijos cuando vienen ya me han contado por WhatsApp, la profesora me ha puesto un cuatro, he suspendido o voy a aprobar o a la hora del patio" (G2).

A pesar de la gran utilidad que en general implican las TIC, los padres manifiestan ciertos temores asociados a su utilización, sobre todo, a partir de la pre-adolescencia (12 años).

"Que se metan en páginas, a mí me ha pasado, o que la niña le mande una foto en bragas, que la coaccionen, chantajes... eso le ha pasado a alguna amiga de mi hija, o que está ligando con un chico de 16 y en realidad tiene 40. Páginas de contacto dan miedo" (G2).

Sobre el bullying coinciden en que es un problema preexistente a las TIC, pero señalan que estas contribuyen a amplificar sus posibles efectos negativos al ser herramientas que fomentan la hiperconexión y permiten un acoso permanente.

La posible dependencia o adicción a la tecnología que puede asociarse al uso intensivo de dispositivos tecnológicos en un hogar hiperconectado, es un temor difuso ya que asumen que sus hijos no dejan de imitar y reproducir sus propios comportamientos y actitudes hacia la tecnología. En este sentido son conscientes de la responsabilidad que implican sus propios comportamientos y actitudes, sobre todo, en edades tempranas.

La percepción de los riesgos descritos no impide que los padres faciliten el acceso de los hijos a los dispositivos tecnológicos motivados por distintas razones:

- por imitación de la 'fiebre digital' de padres y madres: se percibe un mayor uso de dispositivos tecnológicos por parte de los hijos cuyos padres también los usan de forma habitual.

"Porque es lo que ve conmigo, yo tengo una (tablet) y ella tiene la suya y yo creo que es lo que ve, entonces ella me ve manejarla y ella quiere también" (G1).

- como extensión del 'cordón umbilical' más allá del ámbito del hogar: Los móviles permiten la permanente comunicación y control de los hijos. 
"Es verdad que el móvil te puede dar tranquilidad, me dice he salido, o voy a estudiar, ese whatsapp que te pone nos ayuda y estamos más tranquilos" (G2).

- como herramienta escolar para hacer deberes y trabajos, almacenar libros electrónicos y mantener la comunicación con el centro escolar.

"En el colegio ya se lo dan todo por internet, nos han hecho comprar un portátil para trabajar en casa, dependen más de la tecnología" (G1).

- como herramienta de negociación y estímulo para llevar a cabo objetivos educativos como lograr un mejor rendimiento escolar, colaborar en las tareas domésticas, etc., a cambio de acceder a los dispositivos.

"Yo siempre según estudies, según juegas. Tienen sus obligaciones, tienen que hacer su cama, limpiarse... Si tú limpias, tienes todos recogidito, hasta que no lo hagas no juegas. (G2).

- como medio de entretenimiento y distracción en determinadas situaciones en las que padres y madres no pueden dedicar tiempo a entretener y jugar con los hijos.

"Cuando vamos de viaje porque se aburren me lo piden (el móvil) para poner Clan" (G1).

\section{La mensajería instantánea ha generado nuevos canales de comunicación y de in- teracción social en el seno de la familia}

\subsection{Competencias digitales y mediación familiar}

La posible 'brecha digital' entre padres e hijos es prácticamente inexistente en lo que al acceso de dispositivos digitales se refiere, a excepción del manejo de algunos videojuegos para consolas. La brecha tiene más que ver con los distintos usos que hacen padres e hijos de estos dispositivos; lo que condiciona experiencias mediáticas diferentes. Por este motivo, en el ámbito educativo no se percibe que existan barreras tecnológicas que impidan a los primeros ayudar y colaborar en las tareas escolares de los segundos, siendo lo habitual. El problema surge más bien cuando se trata de centros escolares con sistemas educativos bilingües (brecha idiomática). queda solo en casa, se viste, desayuna y se bajan al colegio que está enfrente" (G1).

"Yo me meto en mi Facebook, como están agregados a mi Facebook me puedo meter en el suyo y ver las cosas que tienen" (G2).

"Sí, les reviso las tablets, reviso todo: desde las páginas que se han metido por internet, si han hablado por Facebook..." (G2).

- control de los horarios de uso: más inflexibles y limitados en temporada escolar y más flexibles y amplios en la época estival; pero, en todo caso respetando las horas de reunión familiar que son los desayunos, comidas y cenas. Estas imposiciones de límites en el uso de los móviles no dejan de plantear conflictos y protestas entre los hijos, resolviéndose generalmente con la negociación, sobre todo, del rendimiento escolar.

"Tiene una tablet que le regalamos hace un par de años pero se la restrinjo de viernes cuando llega del colegio hasta el domingo a las 5" (G1).

- control de los tiempos de uso lúdico -2 ó 3 horas al día- e incentivando otras actividades al margen de los dispositivos tecnológicos que fomenten las relaciones personales como pueden ser los juegos de mesa o deportivos.

"También es cierto que nosotros cuando vemos que llevan mucho tiempo con el ordenador, les decimos 'dejadlo', si se han tirado con el ordenador dos horas les decimos que hagan otra cosa. Les dejamos, pero, también les limitamos el tiempo, no es cuestión de que pierdan el tiempo delante de una pantalla" (G1). "Yo normalmente les marco una hora al día, el fin de semana un poco más" (G1).

Otra estrategia de mediación consiste en compartir la navegación. Se trata de una medida de control alternativa, menos jerárquica e invasiva de la intimidad, que implica la vigilancia directa de los contenidos en los dispositivos móviles de adolescentes y preadolescentes. Participando de una misma filosofía, hay padres que comparten con sus hijos la misma clave de acceso o contraseña en todas las aplicaciones y dispositivos del hogar

Para combatir los diferentes miedos relatados con anterioridad, los padres también llevan a cabo otras estrategias que van desde el refuerzo de la comunicación familiar con sus

\begin{abstract}
"Ahora los tienes con los colegios bilingües, el inglés siempre se nos ha dado muy mal y ahora todos los colegios son bilingües. Vienen con el libro de ciencias sociales en inglés y empieza el problema" (G1).
\end{abstract}

En el ámbito de la mediación, los padres llevan a cabo diferentes estrategias de control digital:

- control de los contenidos en los diversos dispositivos así como de los historiales.

"Cuando se acuesta le cotilleo los whatsapps. Sé que es bastante responsable, me voy a trabajar y se



http://wojdylosocialmedia.com/who-consumes-social-media-content 
hijos hasta el trabajo de concienciación del valor e importancia de las imágenes fotográficas y de los vídeos y los posibles peligros que pueden acarrear su publicación en redes o compartirlas en aplicaciones.

"A mi hijo el mayor le hemos concienciado de que eso no se puede hacer, que no puede enviar fotos y lo ve en nosotros dando ejemplo: no hemos subido nunca ninguna foto nuestra; de esa manera ve que no puede subir una foto de él” (G2).

\section{El vínculo intrafamiliar se ve reforzado} por la hiperconexión de la familia, que amplía su radio de acción, y por la inmediatez al permitir una comunicación permanente

\subsection{Nuevos escenarios de comunicación entre pa- dres e hijos vinculados a las TIC}

Aunque se ha indicado previamente la ambivalente percepción que tienen los padres sobre la influencia de las TIC en la comunicación familiar, en términos generales su hegemonía en el entorno familiar no se percibe tanto como un elemento disruptivo de la comunicación en la familia sino más bien como un elemento que ha transformado y creado nuevos canales y espacios de comunicación en el seno de la misma.

Esta transformación de la comunicación familiar se hace más patente cuando los hijos van adquiriendo mayor independencia con respecto a la familia, al comienzo de la ESO.

Las TIC han creado nuevos canales de comunicación intrafamiliar y de interacción social como las conversaciones a dos o grupales por Whatsapp entre los miembros de una familia, donde no sólo se puede conversar sino también compartir fotos y vídeos. El vínculo se ve reforzado por la hiperconexión de la familia, que amplía su radio de acción, y por la inmediatez al permitir una comunicación permanente.

En definitiva, las TIC ayudan a la cohesión familiar siempre y cuando existan buenas relaciones favoreciendo la comunicación familiar más allá de los límites físicos del hogar.

"En mi caso yo creo que se ha transformado, ha cogido varios campos, ya no te limitas a la conversación cara a cara y puedes utilizar el móvil a cualquier hora, la mensajería y no deja de ser comunicación al fin y al cabo y muchas veces es más rápida que si tienes que esperar que lleguen a casa y que te cuenten qué les ha pasado y te lo cuentan en el momento" (G2).

\section{Conclusiones}

Las TIC representan hoy día una tecnología totalmente integrada en la cotidianidad de las familias urbanas de la Comunidad de Madrid llegando a sustituir a la televisión como elemento tecnológico vertebrador y de unión en los hogares.

La 'brecha digital' entre padres e hijos es prácticamente inexistente en el uso y aprendizaje de dispositivos digitales, a excepción del manejo de videoconsolas. No se percibe gran diferencia en el aprendizaje y manejo de dispositivos digitales en general, ni existen barreras tecnológicas que impidan que los padres puedan ayudar y colaborar en las tareas escolares de sus hijos.

De este estudio se desprende también que los hogares urbanos con menores escolarizados tienden a ser hogares hiperconectados debido al número, diversidad y situación dominante de dispositivos tecnológicos. Destacan las tablets y los móviles, seguidos de ordenadores de sobremesa, portátiles y videoconsolas. El único dispositivo que se suele compartir es el ordenador de sobremesa o portátil y su uso se restringe al ámbito profesional, en el caso de los padres, y al educativo en el de los hijos. La utilización del resto de dispositivos es individual.

La opinión de los padres sobre las TIC es muy positiva. Consideran que suponen un avance en el conocimiento y entienden que deben estar integradas en los procesos educativos reglados porque preparan a los niños para que sean capaces de desenvolverse en un futuro laboral cada día más tecnológico.

También aprecian que, a pesar de que las TIC perjudican en ocasiones la comunicación directa entre los miembros de la familia, abren nuevos escenarios de comunicación caracterizados por la hiperconexión y la inmediatez que favorecen la comunicación intrafamiliar, como ocurre con la aplicación Whatsapp. No obstante y aunque su opinión es predominantemente positiva, también manifiestan miedos. Sus principales temores son los riesgos por el uso inadecuado de redes sociales, las páginas de contactos y la posibilidad de que vean contenidos pornográficos.

Coinciden en que el bullying es preexistente a las TIC, pero creen que sus efectos negativos pueden verse incrementados a consecuencia de la hiperconexión, que posibilita un acoso permanente.

Con el objetivo de empoderar a sus hijos frente a los riesgos tecnológicos, los padres median. La mediación además de ser habitual en estos hogares hiperconectados es diversificada. Hay padres que utilizan estrategias de control digital de los contenidos, de los horarios y de los tiempos de uso de los dispositivos accesibles en el hogar. Otros prefieren la navegación compartida y todos ellos establecen estrategias de refuerzo que van desde la intensificación de la comunicación familiar hasta la concienciación de los posibles peligros y repercusiones de la publicación de imágenes y vídeos en redes o en Whatsapp.

\section{Agradecimientos}

Este artículo se enmarca en el proyecto de investigación Auctoritas doméstica, capacitación digital y comunidad de aprendizaje en familias con menores escolarizados, financiado por el Ministerio de Economía y Competititividad (CSO 201342166-R), en el marco del Programa sobre Vulnerabilidad Digital Provuldig (S2015/HUM3434), financiado por la Comunidad de Madrid y el Fondo Social Europeo (2016-2018). 


\section{Bibliografía}

Airebe, Ana; Orozco, Guillermo; Medrano, Concepción (2014). "Family context, television and perceived values. A cross-cultural study with adolescents". Comunicación y sociedad, v. 27, n. 2, pp. 79-99.

http://www.unav.es/fcom/communication-society/es/ resumen.php?art_id $=491$

Álvarez, Miriam; Torres, Alezandra; Rodríguez, Elisa; PadiIla, Sonia; Rodrigo, María-José (2013). “Attitudes and parenting dimensions in parents' regulation of internet use by primary and secondary school children". Computers \& education, v. 67, pp. 69-78.

https://goo.gl/KXAuyd

https://doi.org/10.1016/j.compedu.2013.03.005

Barómetro CIS (2016). Avance de resultados del estudio $n$. 3149. Barómetro de septiembre de 2016.

http://www.cis.es/cis/opencms/ES/NoticiasNovedades/ InfoCIS/2016/Documentacion_3149.html

Bradley, Robert (1995). "Home environment and parenting". En: Bornstein, Marc (ed.). Handbook of parenting, v. 2. Hillsdale, NJ: Earlbaum, pp. 505-530. ISBN: 9780805837797

Bringué-Sala, Xabier; Sádaba-Chalezquer, Charo (2009). La generación interactiva en España. Niños y adolescentes ante las pantallas. Barcelona: Colección Fundación Telefónica, Ariel. ISBN: 9788408091110

http://dadun.unav.edu/handle/10171/17155

Bronffenbrenner, Urie (1987). La ecología del desarrollo humano. Barcelona: Paidós. ISBN: 9788475094441

Callejo, Javier (2001). Investigar las audiencias: un análisis cualitativo. Barcelona: Paidós. ISBN: 9788449310652

Fundación Telefónica (2015). La sociedad de la información en España 2014. Madrid: Ariel. ISBN: 9788408138730 https://goo.gl/NW93/F

García-Jiménez, Antonio (ed.) (2012). Comunicación, infancia y juventud. Situación e investigación en España. UOC: Barcelona. ISBN: 9788490290248

Garmendia-Larrañaga, Maialen; Garitaonandia-Garnacho, Carmelo; Martínez-Fernández, Gemma; Casado-del-Río, Miguel-Ángel (2011). Riesgo y seguridad en internet: Los menores españoles en el contexto europeo. Bilbao: Universidad del País Vasco, EU Kids Online.

https://goo.gl/RnDbmp

Livingstone, Sonia; Bober, Magdalena (2006). "Regulating the Internet al home: Contrasting the perspectives of children and parents". En: Buckingham, David; Willett, Rebekah (eds.). Digital generations: Children, young people, and the new media. Mahwah, NJ: Laurence Erlbaum Associates, pp. 93-113. ISBN: 0805859802

http://eprints.Ise.ac.uk/9013

Livingstone, Sonia; Haddon, Leslie (2008). “Risky experiences for children online: Charting European research on children and the internet". Children \& society, v. 22, n. 4, pp. 314-323.
http://eprints.Ise.ac.uk/27076

https://doi.org/10.1111/j.1099-0860.2008.00157.x

Livingstone, Sonia; Haddon, Leslie; Görzig, Anke; Ólafsson, Kjartan (2011). EU kids online II: Final report. LSE, London: EU Kids Online.

http://eprints.Ise.ac.uk/39351

Martín-Barbero, Jesús (1987). De los medios a las mediaciones. Comunicación, cultura y hegemonía. Méjico: Gustavo Gili. ISBN: 9788476589502

http://perio.unlp.edu.ar/catedras/system/files/de_los_ medios_a_las_mediaciones.pdf

Muñoz-Silva, Alicia (2005). "La familia como contexto de desarrollo infantil. Dimensiones de análisis relevantes para la intervención educativa y social". Portularia, v. 5, n. 2, pp. 147-163. http://rabida.uhu.es/dspace/bitstream/handle/10272/505/ b1518923.pdf?sequence $=1$

Orozco-Gómez, Guillermo (1996). Televisión y audiencias. Un enfoque cualitativo. Madrid: Ediciones de la Torre. ISBN: 9788479601751

Padilla, Sonia; Rodríguez, Elisa; Álvarez, Míriam; Torres, Alezandra; Suárez, Arminda; Rodrigo, María-José (2015). "The influence of the family educational scenario on internet use of primary and secondary school children / La influencia del escenario educativo familiar en el uso de internet en los niños de primaria y secundaria". Infancia y aprendizaje, v. 38, n. 2, pp. 402-434.

https://dialnet.unirioja.es/servlet/articulo?codigo $=5086921$ https://doi.org/10.1080/02103702.2015.1016749

Pérez-Sánchez, Rolando (2013). "Infancia, socialización y TIC". En: Guzmán-Hidalgo, Marta (coord.). Informe Prosic 2013. Hacia la sociedad de la información y el conocimiento. Costa Rica: Prosic, pp. 343-368.

http://www.prosic.ucr.ac.cr/informe-2013

Rodrigo, María-José; Palacios, Jesús (1998). “Conceptos y dimensiones en el análisis evolutivo-educativo de la familia". En: Rodrigo, María-José; Palacios, Jesús (coords.). Familia y desarrollo humano. Madrid: Alianza, pp. 45-50. ISBN: 8420681733

Ruiz-San-Román, José-Antonio; Ortiz-Sobrino, MiguelÁngel; Porto-Pedrosa, Leticia (2013). "Aportaciones de las universidades españolas a la investigación sobre menores y medios de comunicación". Anàlisi: quaderns de comunicación i cultura, pp. 49-64.

Staksrud, Elisabeth; Livingstone, Sonia (2009). "Children and online risk: Powerless victims or resourceful participants". Information, communication and society, v. 12, n. 3 , pp. 364-387.

http://eprints./se.ac.uk/30122

https://doi.org/10.1080/13691180802635455

Valkenburg, Patt; Soeters, Karen (2001). “Children's positive and negative experiences with the internet: An exploratory survey". Communication research, n. 28, pp. 652-675. https://goo.gl/rxT8iT https://doi.org/10.1177/009365001028005004 
$7^{a}$ Conferencia internacional sobre revistas de ciencias sociales y humanidades

CRECS 2017

Cuenca, 4-5 mayo

- El profesional de la
Universidad de

CastillarLa Mancha

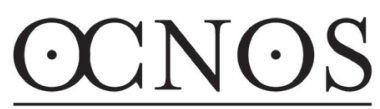

Revista de Estudios sobre Lectura

Think

Cemerald

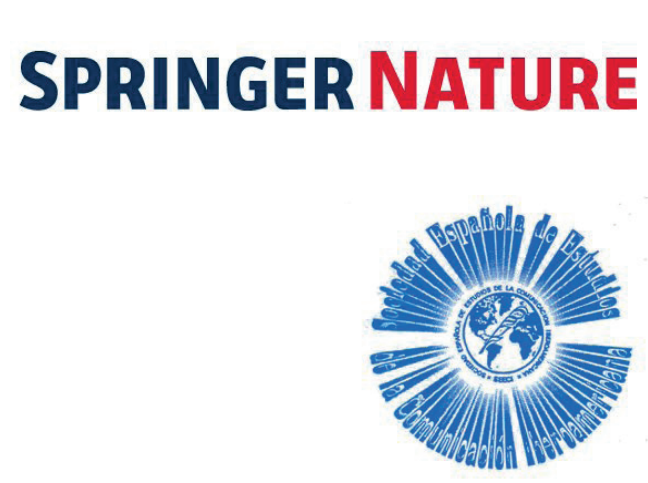

casalinilibri 胥| OEC3metrics.
- Fundación Dialnet UNIVERSIDAD DE LA RIOJA

EBSCO 